\title{
Umbilically and Peripherally Inserted Thin Central Venous Catheters Have Similar Risks of Complications in Very Low Birth Weight Infants
}

\section{S Salonen ( $\nabla$ sanna.salonen@tuni.fi )}

Tampere University: Tampereen Yliopisto https://orcid.org/0000-0002-0758-3419

\section{O Tammela}

Tampere University Hospital: Tampereen yliopistollinen sairaala

\section{AM Koivisto}

Tampere University: Tampereen Yliopisto

\section{P Korhonen}

Tampere University Hospital: Tampereen yliopistollinen sairaala

\section{Research Article}

Keywords: catheter complication, catheter infection, peripherally inserted central catheter, thin umbilical catheter, very low birth weight infant

Posted Date: October 13th, 2021

DOI: https://doi.org/10.21203/rs.3.rs-964597/v1

License: (c) (i) This work is licensed under a Creative Commons Attribution 4.0 International License. Read Full License 
1 Umbilically and peripherally inserted thin central

2 venous catheters have similar risks of

3 complications in very low birth weight infants

4

6

7 Salonen S, B.M. ${ }^{1}$, Tammela O, MD, $\mathrm{PhD}^{2}$, Koivisto $\mathrm{AM}, \mathrm{MSc}^{3}$, Korhonen $\mathrm{P}, \mathrm{MD}, \mathrm{PhD}^{2}$

12 1) Faculty of Medicine and Health Technology, Tampere University, Tampere, Finland

13 2) Tampere Center for Child Adolescent and Maternal Health Research, Faculty of Medicine

14 and Health Technology, Tampere University and University Hospital, Tampere, Finland

15 3) Faculty of Social Science, Health Science, Tampere University, Tampere, Finland

17 Short title: Catheter complications in very low birth weight infants

18 Corresponding author: S. Salonen, Tel +358 44 0410961, sanna.salonen@tuni.fi 


\section{Abstract.}

In order to compare the complications associated with thin (1-2F) umbilical vein catheters

25 (tUVC) and peripherally inserted central catheters (PICC) in very low birth weight (VLBW)

26 infants, the frequencies and causes of the non-elective removal of first tUVCs and PICCs

27 were evaluated in 195 VLBW infants born in one hospital. Data were collected from patient

28 records. Risk factors for catheter complications were analysed using logistic regression

29 analysis. Altogether, 92 (47\%) infants had tUVCs, and 103 (53\%) PICCs. In total, 78 (40\%)

30 infants underwent the non-elective removal of their first catheters, most commonly due to

31 suspected infection $(n=42)$ or catheter dislocation $(n=30)$. Infants with complications had

32 lower birth weights and gestational ages than those without. The frequencies and causes of

33 the non-elective catheter removal were similar in the UUVC and PICC groups. True catheter

34 infection was diagnosed in 31 infants. The number of infections/1,000 catheter days was

35 higher in the tUVC than in the PICC group. In a multivariable analysis, gestational age was

36 found to be associated with catheter infection, but catheter type was not.

37 Conclusion: The odds of catheter complications seem to decrease with increasing

38 gestational age, but no clear association with thin catheter type was found.

40 Key words: catheter complication, catheter infection, peripherally inserted central catheter,

41 thin umbilical catheter, very low birth weight infant

\section{Introduction}

46 Very low birth weight (VLBW) infants require time before beginning enteral feeding, and

47 parenteral nutrition may be required for many weeks. Central venous catheters (CVCs)

48 ensure the administration of sufficient parenteral nutrition, fluids and medication. Peripherally

49 inserted central catheters (PICCs) are thin and can be inserted and removed without

50 anesthesia, but the risk of complications, associated with prolonged hospitalisation, morbidity

51 and even death (1), exists. 
53 By using the umbilical vein as a catheter route, painful skin penetration, which

54 may be associated with an increased risk of infection, can be avoided. The increased risk of

55 complications, especially thrombosis, associated with traditional (3,5-5 F) umbilical venous

56 catheters inhibits their long-term use (2).

58 Several studies have found no differences between PICCs and traditional umbilical venous

59 catheters (UVCs) in terms of general complication $(3,4)$ or catheter infection rates $(5,6)$, but

60 one study found highest CVC-associated infection rates with umbilical catheters (7).

61 Peripherally inserted central catheters and thin (2F) umbilically inserted silicone central

62 venous catheters (tUVC) seem to pose an equal risk of thrombosis, catheter-related sepsis

63 and obstruction (8). More information is needed regarding their use in VLBW infants.

65 Here, the aim was to investigate 1.) the frequency and type of complications in the first thin

66 CVCs, 2.) the differences in catheter complications between tUVCs and PICCs and 3.)

67 the predictors of catheter-related complications, especially catheter infections in VLBW 68 infants.

\section{Subjects and methods}

72 The population consisted of 232 VLBW infants (birth weight $1,500 \mathrm{~g}$ or below) who were born

73 in Tampere University Hospital, during the years 2011-2016 and had received a tUVC or

74 a PICC as the first CVC. Infants who were transferred to another hospital $(N=19)$ or died

$75(\mathrm{~N}=18)$ before removal of the first CVC were excluded. To enable a comparison between

76 tUVCs and PICCs, only the first CVC episode was analysed. The final population included

77195 infants (Figure 1). No significant differences were detected between included and

78 excluded infants in terms of birth weight or gestational age (data not shown). The study was

79 approved by the ethical committee of Pirkanmaa Hospital District (registration ID=R19095R).

81 Information was collected from patient records (Table 1). Chorioamnionitis was considered to

82 be an obstetric clinical diagnosis, as defined previously (9). Infants with a birth weight below 
83 the $10^{\text {th }}$ percentile, adjusted for gestational age, were considered small for gestational age

84 (SGA) $(10,11)$. A diagnosis of necrotising enterocolitis (NEC) was made according to the

85 Bell criteria (at least stage 2) or intraoperative biopsy (12).

87 A PICC or tUVC was inserted if the expected duration of parenteral nutrition was at

88 least one week. It was usually removed when enteral feeding of $130 \mathrm{ml} / \mathrm{kg} / \mathrm{day}$ was reached.

89 After removal, the catheter tip was sent for bacterial culture. During the study period, tUVC

90 was preferred as the first CVC in VLBW infants over PICC. The UVC was inserted as

91 described by Haumont (8) and covered with gauze and a transparent medical dressing. The

92 PICC was usually inserted into a cubital vein using an introducer and then covered with a

93 transparent medical dressing. The catheter tip position was verified by X-rays with contrast

94 media, with the optimal position being extracardial in the central veins.

96 Aseptic principles were followed in the handling of the catheters. Checklists for the insertion

97 and maintenance of the catheters were implemented during 2015, and since then, the

98 practice has been to remove or change thin CVCs after 14 days' dwell time if possible. A

99 multidisciplinary group supervised the hygienic procedures on the ward.

101 Our primary outcome measure was catheter complication, defined as non-elective catheter 102 removal for a catheter-related reason more than 24 hours after insertion. Complications were

103 classified as follows: suspected infection, dislocation and other complications

104 (disconnected/blocked/broken).

105

106 In the subgroup analysis of catheter infections, we used two time periods: 1) within one day

107 before and one day after catheter removal and 2) within three days before and three days

108 after catheter removal. The diagnostic criteria for true catheter infection included a positive

109 blood culture without any other focus, as well as the symptoms and signs of infection, such

110 as temperature instability, hypotension, apnea, increased need for oxygen and/or ventilatory

111 support, heart rate abnormalities, feeding intolerance, lethargy, irritability, skin lesions and at

112 least one of the following laboratory parameters: white blood cell count $<5$ or $>30 x$ 
$11310^{9}$ cells $/ \mathrm{L}$, immature to total neutrophil ratio $>0.2$, absolute neutrophil count $<1000 / \mu \mathrm{L}$,

114 platelet count $<100 \times 10^{9} / \mathrm{L}, \mathrm{CRP}>15 \mathrm{mg} / \mathrm{L}$, glucose intolerance or hypoglycemia, acidosis

115 (modified from $(13,14)$ ).

116

117 Statistical analysis

118 Group comparisons with categorical variables were performed using crosstabulation and the

119 Chi-square or Fisher's exact test, and those with continuous variables were performed using

120 a Mann-Whitney U-test. Differences between catheter types in terms of the frequencies of

121 complications and true catheter infections were also analysed per 1,000 catheter days using

122 the OpenEpi program's Mid-p procedure.

124 Risk factors were analysed using univariable and multivariable logistic regression analysis

125 separately for 1) any catheter complication and 2) true catheter infection diagnosed within

126 three days before or after catheter removal. The independent variables were gestational age

127 at birth (weeks), SGA (no/yes), gender (female/male), mode of delivery (vaginal/cesarean

128 section), need for mechanical ventilation (no/yes) and central venous catheter type

129 (tUVC/PICC). Gestational age and ventilator therapy were included in the models based on

130 the results of univariable analyses, SGA to take into account birth weight, and gender and

131 mode of delivery as known factors affecting neonatal outcomes.

133 P-values $<0.05$ (two-sided) were considered to be statistically significant. Statistical analyses

134 were performed using SPSS (Versions 24 and 25).

\section{Results}

138 Catheter complications

139 Altogether, 92 (47\%) infants had a tUVC and 103 (53\%) infants a PICC. In total 78 (40\%)

140 infants underwent non-elective removal of their first catheter. Of these, 42 (54\%) were

141 removed for suspected infection, $30(38 \%)$ for dislocation and six $(7.8 \%)$ for other reasons 
142 (three disconnected, two broken and one obstruction) (Figure 1). The median catheter dwell

143 time at non-elective removal was 10 days.

145 Clinical characteristics of the infants are presented in Table 1. Infants who had catheter

146 complications in their first catheter had lower birth weights, were born more premature and

147 needed ventilator therapy more commonly and for longer periods.

149 Comparison between catheter types

150 The comparisons between infants with tUVCs and those with PICCs are presented in Table

151 2. Umbilical catheters were inserted at an earlier age than PICCs (md(range) 0 (0-5) vs 2 (0-

15232 ) days, $p<0.001$. The most common reason for the non-elective removal of tUVCs was

153 suspected infection and dislocation for PICCs. No significant difference was found in the total

154 catheter complication rate between infants with tUVCs and those with a PICC. True catheter

155 infection within +/-3 days from catheter removal was more common in the tUVC group than in

156 the PICC group, but the groups did not differ in terms of the $+/-1$ day criteria. The occurrence

157 of NEC was similar between the groups (tUVC 12 (13.0\%) vs PICC 14 (13.6\%), p=0.910).

159 Ten infants died during their hospital stays after the removal of the first catheter. There were

160 more deaths in the tUVC group than the PICC group (8(8.7\%) vs. $2(1.9 \%), p=0.033)$. In two

161 tUVC cases, the cause of death was catheter sepsis. Eight deaths did not have catheter-

162 related causes and occurred 4-44 days after the removal of the first catheter.

164 Risk factors for catheter complications and catheter infections

165 The results of risk factor analyses of catheter complications generally are presented in Table

166 3. Low gestational age and ventilator therapy were associated to catheter complication in the

167 univariable analysis. In the multivariable logistic regression analysis, gestational age

168 was marginally significantly associated with catheter complications generally, with an OR

$169(95 \% \mathrm{Cl})$ of $0.822(0.675-1.000), p=0.05$, but ventilator therapy was not. 
171 Altogether, 21 (10.8\%, 8.4/1000 catheter days) infants had true catheter infection (positive

172 blood culture combined with symptoms) within $+/-1$ day of catheter removal, and 31 (15.9\%,

173 12.3/1000 catheter days) infants had such within $+/-3$ days of catheter removal. Among the

174 latter, 26 catheters were removed due to suspected infection, one due to catheter

175 obstruction, and four electively. In these five cases, signs and symptoms of

176 infection developed within three days after catheter removal.

178 In the univariable analysis, lower gestational age and ventilator therapy were associated with 179 true catheter infection within 3 days before or after catheter removal, but in the multivariable 180 logistic regression analysis only gestational age remained significant risk factor. No 181 association was found between catheter infection and CVC type. (Table 3)

183 The most common pathogen in both blood cultures and catheter tip cultures was coagulase184 negative staphylococcus (CoNS). Tip culture findings were available from 139 (71.3\%) 185 catheters. Of these, $62(44.6 \%)$ revealed pathogen growth, including $30(35 \%)$ of the 186 electively removed catheters and seven that were removed due to a dislocation. The tip 187 culture was positive in 19 of $31(61.3 \%)$ catheters in the true catheter infection group, and in $18813(41.9 \%)$, the culture revealed the same bacteria in both the blood and catheter tip.

190 Discussion

192 In this study of VLBW infants, non-elective removals of thin umbilical CVCs and the first

193 peripherally inserted CVCs were common. Nearly half of the removals were due to

194 suspected infection. We did not find significant differences between total complication rates

195 with the use of either tUVCs or PICCs, but non-elective removals due to suspected

196 infection were more common in the tUVC group. Two infants with tUVCs died due to catheter

197 infection. Also, the rate of infections/1,000 catheter days was higher in the tUVC group than

198 the PICC group. However, in the multivariable logistic regression analysis, the only

199 statistically significant risk factor for true catheter infection was low gestational age. 
201 In the earlier studies non-elective PICC removal rates in neonates $(3,15-17)$ or infants (1)

202 have varied from $9.97 \%$ to $37.2 \%$. In our study, a high percentage (40\%) of the first thin

203 CVCs (PICC or tUVC) were removed non-electively. Differences in patient populations and

204 the definitions of complications mostly account for differences between studies. We did not

205 find any significant differences between the overall complication rates of the tUVC or PICC.

206 Previous studies have investigated mainly traditional, uncovered UVC catheters and had

207 different study design so they are not comparable with our study. $(4,7,9,11)$ To our

208 knowledge, this study is one of the few focusing on premature VLBW infants and the use of 209 tUVCs,

211 As in prior studies $(1,15,17)$, infants with any catheter complications had lower birth

212 weights and gestational ages than those without complications. They were also more

213 commonly and lengthily exposed to invasive treatments, including ventilator therapy and

214 parenteral nutrition. We focused on the complications regarding the first thin CVCs, and no

215 strict conclusions can be drawn regarding their role in the development of later problems,

216 such as NEC.

218 In our population infants with tUVC were lighter and more premature at birth than infants with

219 PICC, which might contribute to the differences in infections and death rates. The greater

220 percentage of deaths in the tUVC group was probably not associated with the use of tUVCs.

221 Multivariable logistic regression analysis did not reveal any association between catheter

222 type and the risk of either any catheter complication or catheter infection. Thin UVC has

223 some benefits compared to PICC, because it can be inserted pain free, without penetrating

224 the skin of a fragile infant. When tUVC is used as the first CVC, the peripheral veins remain

225 intact for later use, which is beneficial, especially when the need for prolonged parenteral

226 nutrition is likely.

228 Our definitions of catheter related sepsis were based on positive blood cultures and 229 symptoms and laboratory findings compatible with infection within one or three days before 230 and after catheter removal. The removal of CVCs has been suggested to induce bacteremia, 
231 occurring within 72 hours after catheter removal, even in $23 \%$ of the sepsis cases (18). We

232 included this wider timeframe to ensure that no catheter related infections would be

233 missed. The shorter timeframe is in accordance with Center for Disease Control and

234 Prevention criteria (19).

235

236 Previous studies have reported sepsis rates of $1.66-18.1 / 1,000$ catheter days $(5-7,20,21)$.

237 Our infection rates were within this range. The various hygienic practices of the units, ward

238 patient loads and patient case mixes help explain the differences between studies. According

239 to our logistic regression analysis, the most important risk factor for catheter infections

240 was low gestational age, as reported previously $(15,20)$. An increased need for invasive

241 treatments, fragile skin barriers and immature immune systems contribute to an increased

242 risk for infections (22) in very low gestational age infants.

244 In line with previous studies, CONS was the most common pathogen found in the blood $(2,6$,

$24520,21,23)$ and catheter tip cultures (15). Asymptomatic bacterial contamination of the

246 catheters were common (24). The use of catheter tip cultures as diagnostic criteria for

247 catheter related sepsis is challenging. The infant may have received several antibiotic doses,

248 sometimes to the CVC, before the removal of the catheter, which may bias the culture

249 results.

250

251 The limitations of this study were its retrospective design and relatively small sample size.

252 However, our results add knowledge about CVC complications in VLBW infants. The

253 catheter-related sepsis classification we used was clinically applicable. Because all infants

254 were treated in one unit, we had access to all clinical details in the patient records. Treatment

255 practices did not differ markedly between patients.

257 Conclusion

259 Gestational age was associated with both central venous catheter complications and

260 infections in VLBW infants. No clear association was found between central venous catheter 
type and catheter complications. Our data suggest that neither tUVCs nor PICCs seem to be

262 superior regarding complication risk.

\section{References}

1. Costa P, Kimura AF, Brandon DH, Damiani LP. Predictors of Nonelective Removal of

268 Peripherally Inserted Central Catheters in Infants. Biol Res Nurs. 2016 Mar;18(2):173-80.

269 2. Ramasethu J. Complications of vascular catheters in the neonatal intensive care unit. Clin

270 Perinatol. 2008 Mar;35(1):199,222, x.

271 3. Dongara AR, Patel DV, Nimbalkar SM, Potana N, Nimbalkar AS. Umbilical Venous

272 Catheter Versus Peripherally Inserted Central Catheter in Neonates: A Randomized

273 Controlled Trial. J Trop Pediatr. 2017 Oct 1;63(5):374-9.

274 4. Konstantinidi A, Sokou R, Panagiotounakou P, Lampridou M, Parastatidou S, Tsantila K, 275 et al. Umbilical Venous Catheters and Peripherally Inserted Central Catheters: Are They

276 Equally Safe in VLBW Infants? A Non-Randomized Single Center Study. Medicina (Kaunas).

2772019 Aug 6;55(8):10.3390/medicina55080442.

278 5. Shalabi M, Adel M, Yoon E, Aziz K, Lee S, Shah PS, et al. Risk of Infection Using

279 Peripherally Inserted Central and Umbilical Catheters in Preterm Neonates. Pediatrics. 2015

280 Dec;136(6):1073-9.

281 6. Dubbink-Verheij GH, Bekker V, Pelsma ICM, van Zwet EW, Smits-Wintjens, V. E. H. J., 282 Steggerda SJ, et al. Bloodstream Infection Incidence of Different Central Venous Catheters 283 in Neonates: A Descriptive Cohort Study. Front Pediatr. 2017 Jun 20;5:142.

284 7. Yumani DF, van den Dungen, F. A., van Weissenbruch MM. Incidence and risk factors for 285 catheter-associated bloodstream infections in neonatal intensive care. Acta Paediatr. 2013 286 Jul;102(7):293.

287 8. Haumont D, de Beauregard VG, Van Herreweghe I, Delanghe G, Ciardelli R, Haelterman 288 E. A new technique for transumbilical insertion of central venous silicone catheters in 289 newborn infants. Acta Paediatr. 2008 Jul;97(7):988-90.

290 9. Newton ER. Chorioamnionitis and intraamniotic infection. Clin Obstet Gynecol. 1993 291 Dec;36(4):795-808.

292 10. Sankilampi U, Hannila ML, Saari A, Gissler M, Dunkel L. New population-based 293 references for birth weight, length, and head circumference in singletons and twins from 23 294 to 43 gestation weeks. Ann Med. 2013 Sep;45(5-6):446-54.

295 11. Schlaudecker EP, Munoz FM, Bardaji A, Boghossian NS, Khalil A, Mousa H, et al. Small 296 for gestational age: Case definition \& guidelines for data collection, analysis, and 297 presentation of maternal immunisation safety data. Vaccine. 2017 Dec 4;35(48 Pt A):651829828. 
12. Bell MJ, Ternberg JL, Feigin RD, Keating JP, Marshall R, Barton L, et al. Neonatal necrotizing enterocolitis. Therapeutic decisions based upon clinical staging. Ann Surg. 1978 Jan;187(1):1-7.

13. Puopolo KM, Benitz WE, Zaoutis TE, COMMITTEE ON FETUS AND NEWBORN, COMMITTEE ON INFECTIOUS DISEASES. Management of Neonates Born at $\geq 35$ 0/7 Weeks' Gestation With Suspected or Proven Early-Onset Bacterial Sepsis. Pediatrics. 2018 Dec;142(6):e20182894. doi: 10.1542/peds.2018-2894.

14. Ronchetti MP, Bersani I, Piersigilli F, Auriti C. Neonatal sepsis. Arch Paediatr Dev Pathol. 2017:1(3): 1015.

15. Wen J, Yu Q, Chen H, Chen N, Huang S, Cai W. Peripherally inserted central venous catheter-associated complications exert negative effects on body weight gain in neonatal intensive care units. Asia Pac J Clin Nutr. 2017 Jan;26(1):1-5.

16. Goldwasser B, Baia C, Kim M, Taragin BH, Angert RM. Non-central peripherally inserted central catheters in neonatal intensive care: complication rates and longevity of catheters relative to tip position. Pediatr Radiol. 2017 Nov;47(12):1676-81.

17. Beard L, Levek C, Hwang S, Grover T. Prediction of Nonelective Central Venous Catheter Removal in Medically Complex Neonates. Pediatr Qual Saf. 2019 Aug 5;4(4):e179.

18. van den Hoogen A, Brouwer MJ, Gerards LJ, Fleer A, Krediet TG. Removal of percutaneously inserted central venous catheters in neonates is associated with the occurrence of sepsis. Acta Paediatr. 2008 Sep;97(9):1250-2.

19. Bloodstream Infection Event (Central Line-Associated Bloodstream Infection and Noncentral Line Associated Bloodstream Infection) [Internet].; 2021 [updated Jan,; cited 2.3.2021].

20. Sanderson E, Yeo KT, Wang AY, Callander I, Bajuk B, Bolisetty S, et al. Dwell time and risk of central-line-associated bloodstream infection in neonates. J Hosp Infect. 2017 Nov;97(3):267-74.

21. Milstone AM, Reich NG, Advani S, Yuan G, Bryant K, Coffin SE, et al. Catheter dwell time and CLABSIs in neonates with PICCs: a multicenter cohort study. Pediatrics. 2013 $\operatorname{Dec} ; 132(6): 1609$.

22. Melville JM, Moss TJ. The immune consequences of preterm birth. Front Neurosci. 2013 May $21 ; 7: 79$.

23. D Cartwright. Central venous lines in neonates: a study of 2186 catheters. Arch Dis Child Fetal Neonatal Ed. 2004 Nov,:89(6): F504-F508.

24. Sobczak A, Klepacka J, Amrom D, Żak I, Kruczek P, Kwinta P. Umbilical catheters as vectors for generalized bacterial infection in premature infants regardless of antibiotic use. $J$ Med Microbiol. 2019 Sep;68(9):1306-13. 
Figures

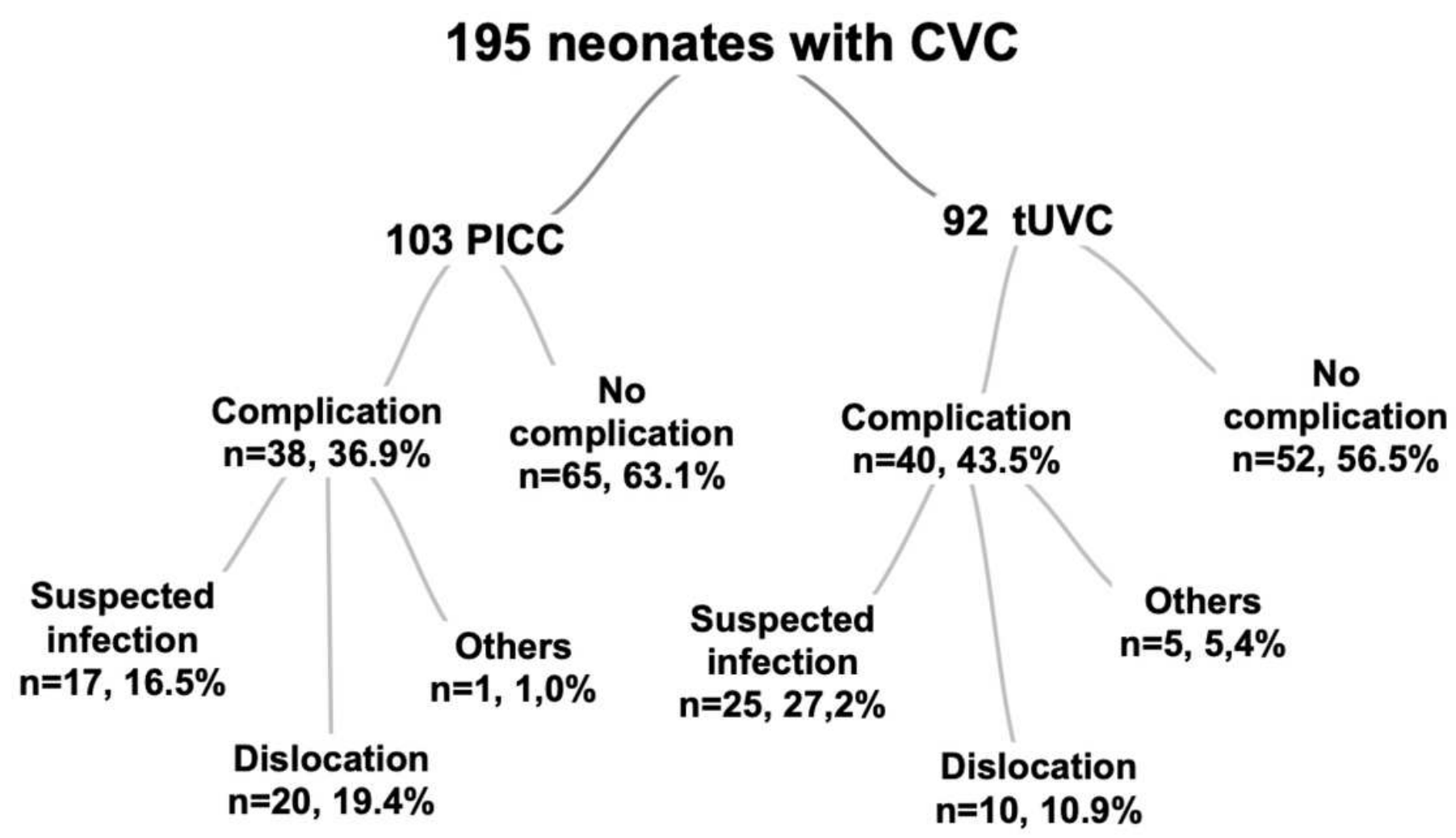

Figure 1

Study population, $\mathrm{CVC}=$ central venous catheter, $\mathrm{PICC}=$ peripheral inserted central catheter, $\mathrm{UUVC}=$ thin $(1-$ $2 \mathrm{~F})$ umbilically inserted central venous catheter. Complication was defined as a non-elective removal of the catheter

\section{Supplementary Files}

This is a list of supplementary files associated with this preprint. Click to download.

- Table1.pdf

- Table2.pdf

- Table3.pdf 